\title{
Wieder Hausarzt nach einer schweren Lebenskrise
}

\author{
Autor: ein betroffener Kollege, Co-Autorin: Mirjam Tannera \\ ${ }^{a}$ Dr. med., Fachärztin für Psychiatrie und Psychotherapie, Leitungsausschuss ReMed
}

Nach einer schweren privaten und beruflichen Krise findet ein engagierter Hausarzt wieder zurück in den Beruf. Er berichtet hier, wie er durch die Begleitung von ReMed, dem Unterstützungsnetzwerk für Ärzte, wieder Vertrauen gewonnen hat. Mit dem neuen Projekt «Praxispraktikum bei einem Mentorarzt» bietet ReMed Ärzten, die nach einer Krise wieder in den Berufsalltag einsteigen möchten, eine zusätzliche Hilfe an.

\begin{abstract}
"Aufgrund einer schweren Erschöpfungsdepression war ich nicht mehr in der Lage, meinen Beruf als Hausarzt auszuüben. Ich verlor nicht nur meinen erfüllenden Alltag als Landarzt, sondern durch die Trennung von der Familie auch meine Kinder, mein Zuhause. Dadurch zerbrach mein berufliches und privates Lebensfundament.
\end{abstract}

In den Gesprächen mit ReMed ging es um eine stützende Beratung und auch darum, eine berufliche Perspektive zu erarbeiten.

Hilfe erhielt ich unter anderem von ReMed in Form von periodischen Telefongesprächen. In diesen ging es zuerst um eine stützende Beratung und schliesslich

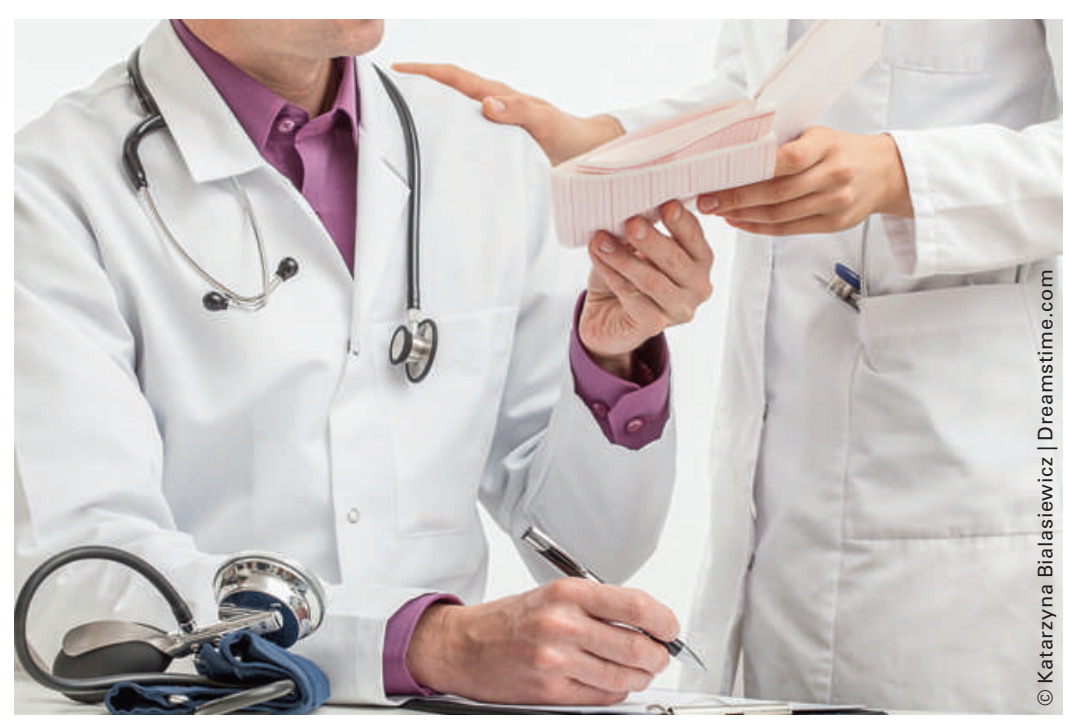

Gut begleitet zurück in den Arztberuf: Mit einem Praktikum bei einem Mentor-Kollegen, einer Mentor-Kollegin.

\section{ReMed ist für Sie da}

Benötigen Sie oder jemand aus Ihrem Umfeld professionelle Hilfe? Wenden Sie sich an ReMed: Das Unterstützungsnetzwerk für Ärztinnen und Ärzte respektiert das Arztgeheimnis und berät Sie kompetent. ReMed begleitet Sie in beruflichen und persönlichen Krisen. Auf Ihre Kontaktnahme meldet sich das Beraterteam innerhalb von 72 Stunden zurück. Weitere Informationen und Kontakt: www.swiss-remed.ch, help[at]swiss-remed.ch, Tel. 0800073633 .

auch darum, eine berufliche Perspektive zu erarbeiten und den Wiedereinstieg in meinen Hausarztberuf zu planen. Finanziell unterstützte mich die Schweizerische Ärzte-Krankenkasse SAEKK mit Taggeldzahlungen, was eine grosse Erleichterung war. Dennoch belastete mich die chronische Arbeitslosigkeit respektive Arbeitsunfähigkeit sehr und führte dazu, dass ich wiederholt in Einsamkeit und Depressionen versank. Die bevorstehende Scheidung, die Trennung von den Kindern und fehlende soziale Kontakte am neuen Wohnort machten mir zusätzlich zu schaffen. Obwohl ich in dieser Zeit nicht fähig gewesen wäre, als Hausarzt zu arbeiten, fehlte mir diese erfüllende Aufgabe.

\section{Abgestempelt und chancenlos}

Im Frühling 2010 fühlte ich mich ein wenig besser. Ich bewarb mich für Arbeitsstellen bei Versicherungen und vor allem bei Hausärzten mit der Bitte, im Sinne eines beruflichen Wiedereinstiegs in ihrer Praxis mitarbeiten zu können. Es war sehr frustrierend, dass die meisten Praktiker eine Anstellung ablehnten. Die Tatsache, dass ich aus gesundheitlichen Gründen ein Jahr nicht gearbeitet hatte, bewerteten sie offenbar als 
zu grosses Risiko. Das Misstrauen, das mir entgegenschlug, nagte an meiner Selbstachtung und ich fühlte mich als krank abgestempelt.

Die fehlende Arbeit und Tagesstruktur und die berufliche und soziale Isolation haben mich ein halbes Jahr später erneut in eine Depression getrieben, die eine stationäre Behandlung erforderte. Nachdem eine Er-

\section{Mit der Zeit betreute ich die Patienten zunehmend selbständig. Meine Ängste - ins- besondere nicht zu genügen - verflogen.}

krankung medikamentös und psychotherapeutisch erfolgreich behandelt wurde, ging es mir endlich wieder besser. Ich begann mich $\mathrm{zu}$ bewerben und hatte nach insgesamt zwei Jahren Erwerbsunfähigkeit Glück: Ein bekannter Arztkollege bot mir an, in seiner Praxis zu 50\% mitzuarbeiten. Dies geschah im Sinne eines "Arbeitsversuches" und wurde von der Schweizerischen Ärzte-Krankenkasse mitfinanziert.

\section{«Wiedereinsteiger-Praxispraktika»: Pilotprojekt sucht Mentorärzte und Wiedereinsteiger}

Kollegen können nach einem krisenbedingten Ausfall vor dem Wiedereinstieg in die eigene Praxis verunsichert sein. Mit einem Praxispraktikum bei einer Mentorärztin, einem Mentorarzt können sie wieder Sicherheit und Selbstbewusstsein für die verantwortungsvolle Praxistätigkeit gewinnen. In einem mehrwöchigen Praktikum arbeitet der Arzt unter der Supervision des Mentors, gewinnt wieder Vertrauen in seine Kompetenz und ortet allfällige Lücken in seiner medizinischen, sozialen und Praxisführungs-Kompetenz.

Die Vorteile einer solchen Kooperation für die Mentorärzte liegen angesichts des wachsenden Mangels an Grundversorgern in möglichen Praxisvertretungen und Praxisübergaben sowie in der Qualitätsförderung und Qualifikation des Betriebs. Mentoren haben idealerweise Erfahrung als Lehrärzte und sind erfahrene niedergelassene Ärzte, die mit dem Angebot von ReMed vertraut sind.

Suchen Sie einen niedergelassenen Arzt, bei dem Sie ein solches Wiedereinstiegspraktikum machen können? Oder sind Sie interessiert daran, als Mentorarzt einem Kollegen den Wiedereinstieg zu ermöglichen? Befristet bis Ende 2017 läuft eine Pilotphase. Sind Sie interessiert oder betroffen? Dann melden Sie sich jetzt via info[at]swiss-remed.ch oder 0313591200.
Intervisionsgruppen: Termine 2016

ReMed initiierte 2009 kollegiale Intervisionen, auch auf Wunsch von Kolleginnen und Kollegen. Seither organisiert das Unterstützungsnetzwerk regelmässig Peer-Groups (6-10 Teilnehmer, 2-3-mal/Jahr). Die Teilnehmenden erarbeiten gemeinsam ihre Fallfragen zu Mentoring, Coaching, Beratung, Therapie und anderen Aspekten kollegialer Begleitung (juristisch, versicherungsrechtlich usw.). Setzen Sie sich mit uns in Verbindung, nehmen Sie an einer Sitzung teil und lernen Sie unsere Arbeit kennen. Kontakt: Peter Birchler, Tel. 0443420910 oder peter.birchler[at]hin.ch

Nächste Daten:

27.10.16 Hotel Plaza Zürich 14-18 Uhr

17.11.16 Hotel Plaza Zürich 14-18 Uhr

\section{Behutsam zurück in den Beruf}

Zu Beginn betreute ich die Patientinnen und Patienten unter Aufsicht des Praxisinhabers, mit der Zeit zunehmend selbständig. Meine Ängste - insbesondere nicht zu genügen - verflogen. Nach etwa zwei Monaten fühlte ich mich fachlich sowie menschlich kompetent und bereit, mich für weitere Stellen zu bewerben. Ich fand eine 70-Prozent-Stelle als Allgemeinmediziner in einer grösseren städtischen Notfall-Praxis. Mit anfänglicher Unterstützung dieses Betriebes erlebte ich dankbar eine umfassende Reintegration in meinen Beruf als Allgemein- und Hausarzt.

Es liegt mir sehr am Herzen, dass Kolleginnen und Kollegen, die sich in einer schweren Lebenszeit befinden, frühzeitig Hilfe erhalten. Wesentlich ist zudem, dass sie die Chance bekommen, auch nach längerer Krankheit wieder in ihren Beruf zurückzukehren, sofern sie das wirklich wollen. Dabei scheint mir ausserordentlich wichtig, dass der berufliche Wiedereinstieg in den Händen unseres Berufstandes bleibt - und nicht über die langwierigen, unpersönlichen, kostenproduzierenden und kaum berufsbezogenen Wege der Invalidenversicherung laufen soll. Ich denke, wir sollten uns nicht nur unseren Patientinnen und Patienten gegenüber solidarisch zeigen, sondern ebenso gegenüber unseren Berufskollegen, die sich in einer Notsituation mit unsicherer persönlicher und beruflicher Zukunft befinden!» 